\title{
Noble-Metal Nanostructures as Artificial Enzymes: Controlled Synthesis and Electron Microscope Characterizations
}

\author{
Xiaohu Xia ${ }^{1}$ and Haihang $\mathrm{Ye}^{1}$ \\ 1. Department of Chemistry, Michigan Technological University, Houghton, MI.
}

As alternatives to natural enzymes, artificial enzyme made of inorganic nanomaterials have received great interest in recent years because of their broad range of applications. One of the most promising examples is artificial peroxidase [1]. While a variety of artificial peroxidase have been actively developed over the past decade, their relatively low catalytic efficiency has not been addressed in the area.

It is well documented that the catalytic activity of nanocrystals for a reaction is highly dependent on the type of crystallographic plane (which has a strong correlation with the shape) and elemental composition on the surface. Taking the oxygen reduction reaction as an example, the catalytic activity of $\mathrm{Pd}$ nanocubes enclosed by $\{100\}$ facets with a square lattice was found to be $\sim 9.4$ times higher than that of $\mathrm{Pd}$ octahedrons encased by $\{111\}$ facets with a hexagonal lattice [2]; A 22 -fold increase in area specific activity was achieved when Pd nanocrystal surface had been coated with a smooth Pt skin of 2-3 atomic layers [3]. Those former studies give us a clue that the catalytic activity of artificial peroxidases may also has a strong dependence on the shape and elemental composition. This assumption forms the basis of our recent research, in which we carefully controlled the structure of noble-metal nanocrystals and substantially enhanced their peroxidase-like catalytic activities.

In our initial study, we have developed a novel type of artificial peroxidase - Pd@ $\operatorname{Ir}_{1-2 \mathrm{~L}}$ core@shell nanocubes - that were synthesized by coating Pd nanocubes with a few atomic layers of Ir (see Figure 1) [4]. Atomic-level crystal structure and elemental composition of the core@shell cubes could be clearly characterized by the high-angle annular dark-field scanning transmission electron microscope (HAADFSTEM) images and X-ray spectroscopy (EDX) analyses. These Pd@ $\operatorname{Ir}_{1-2 \mathrm{~L}}$ cubes showed a record-high catalytic efficiency with $K_{\text {cat }}=10^{6} \mathrm{~s}^{-1}$ ( $K_{\text {cat }}$ is defined as the maximum number of chemical conversions of substrate molecules per second per catalyst), which is approximately 3 orders of magnitudes higher than their natural counterparts. Our theoretical calculations based on density functional theory (DFT) indicated that the ultrathin Ir overlayers on Pd surface is responsible for the substantially enhanced catalytic efficiency because they could increase the adsorption energies of key chemical species in the catalytic reaction.

With this Pd@Ir cubes serving as a starting point, very recently, we have invented several other types of artificial peroxidases with superior properties and excellent performances in biomedical applications. Among them, a notable example is $\mathrm{Au} @ \mathrm{Pt}$ core@shell nanoparticles (see Figure 2) [5]. Au nanoparticles are known to offer highly intense color signals (red, in most cases) owing to an optical phenomenon known as localized surface plasmon resonance (LSPR), making them extremely useful in many applications. Nevertheless, the color intensity of Au nanoparticles is essentially confined by the inherent plasmonic activity. We have demonstrated that such confinement can be broken by coating conventional Au nanoparticles with ultra-thin skins of Pt (i.e., Au@Pt nanoparticles). This is because the Pt skins endowed the nanoparticles with strong peroxidase-like catalytic activities, allowing them to generate intense secondary blue color signal. Significantly, the blue color from catalysis was orders of 
magnitudes stronger than the intrinsic red color from plasmonics of Au nanoparticles.

In conclusion, we have successfully developed a series of metal nanostructures with substantially enhanced enzymatic activities. The key was to carefully engineer the crystal structure and elemental compositions at atomic level, which could be analyzed by high-performance electron microscopes.

\section{References:}

[1] H Wei, E Wang, Chemical Society Reviews 42 (2013), p. 6060.

[2] M Shao, et al, Chemical Communications 47 (2011), p. 6566.

[3] J Park et al, ACS Nano 9 (2015), p. 2635.

[4] X Xia et al, ACS Nano 9 (2015), p. 9994.

[5] Z Gao et al, Nano Letters 17 (2017), p. 5572.
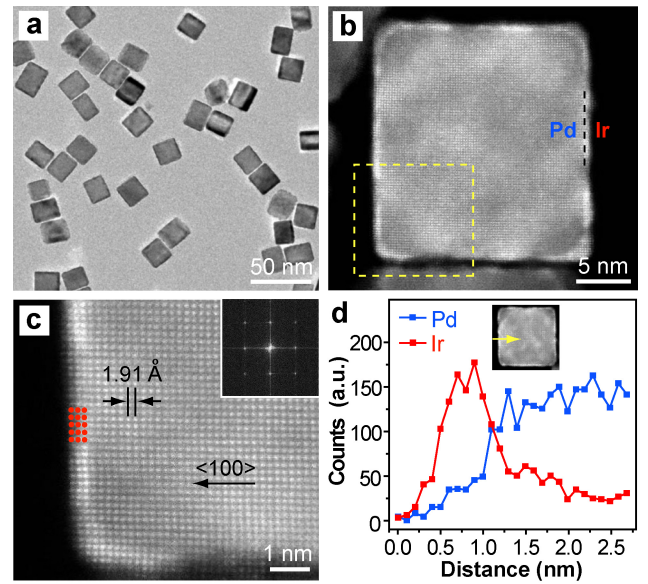

Figure 1. Structural and compositional analyses of Pd@Ir core@shell nanocubes. ${ }^{[4]}$ (a) TEM image; (b) HAADF-STEM image; (c) Atomic-resolution HAADF-STEM image taken from the corner region marked by a yellow box in (b); (d) Line-scan EDX spectra of elemental Pd and Ir.
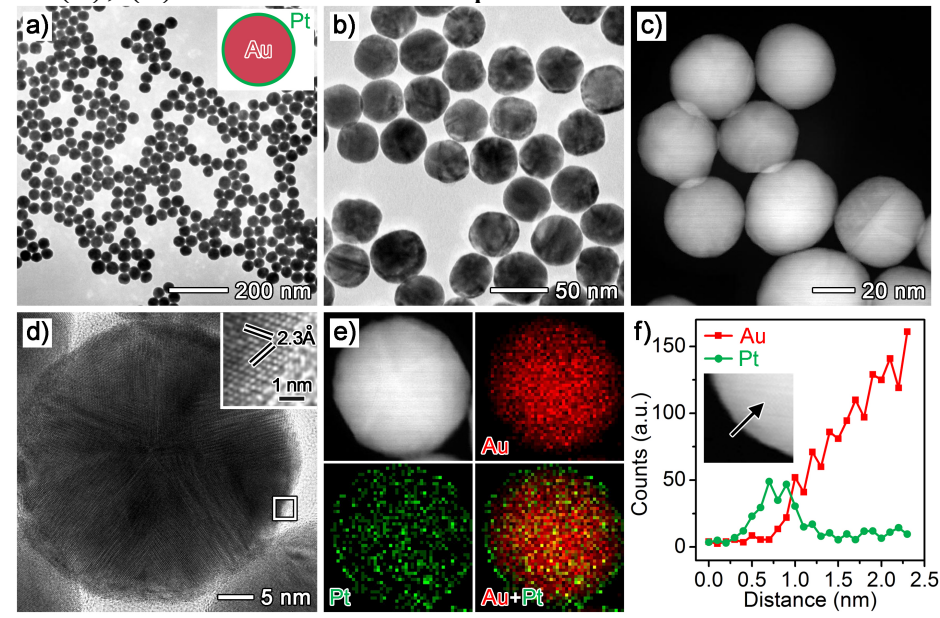

Figure 2. Structural and compositional analyses of Au@Pt core@shell nanoparticles. ${ }^{[5]}$ (a, b) TEM images; (c) HAADF-STEM image; (d) High resolution TEM image; (e) EDX mapping image; (f) Linescan EDX spectra of elemental Au and Pt. 